\title{
artigo
}

Lima, F.N.; Almeida, C.A.P.L.; Campelo, R.C.V.; Lima, L.H.O.; Vilarouca da Silva, A.R.; Machado, A.L.G.;

Prevalência de risco cardiovascular e fatores associados em adolescentes

\section{Prevalência de risco cardiovascular e fatores associados em adolescentes}

\author{
Prevalence of cardiovascular risk and associated factors in adolescents \\ Prevalencia de riesgo cardiovascular y factores asociados en adolescentes
}

\begin{abstract}
RESUMO
Os fatores de riscos cardiovasculares e fatores associados podem em conjunto somar-se para o desenvolvimento de Doenças Cardiovasculares. Objetivo: Analisar a frequência de fatores de risco cardiovascular e fatores associados em adolescentes. Método: Estudo transversal realizado com 251 estudantes do ensino médio da cidade de Teresina (PI), entre 14 a 17 anos de idade. Foram obtidas informações sobre o estilo de vida, dados antropométricos e clínicos. Resultados: A média de idade dos adolescentes de $16,2 \pm 0,76$ anos. Observou-se prevalência de sonolência diurna excessiva $(88 \%)$, fisicamente inativos (38,2\%), pré-hipertensos $(14,7 \%)$ PAS e $(27,1 \%)$ PAD. Sobre o índice de massa corporal o sexo feminino apresentou maiores índices, porém para circunferência da cintura e pescoço o sexo masculino apresentou maiores índices $(p<0,05)$. Conclusão: A presença e associações positivas entre os fatores de riscos que podem contribuir para o desenvolvimento de doenças cardiovasculares servem como sinalizadores para que medidas preventivas sejam tomadas.
\end{abstract}

DESCRITORES: Fatores de Risco; Estilo de Vida; Adolescentes.

\section{ABSTRACT}

Cardiovascular risk factors and associated factors may together add to the development of cardiovascular diseases. Objective: The frequency of cardiovascular risk factors and associated factors in adolescents was analyzed. Method: Cross-sectional study with high school students from the city of Teresina (PI), with a sample of 251 adolescents with a mean age of $16.2 \pm 0.76$ years. Lifestyle information, anthropometric and clinical data were obtained. Results: Adolescents presented prevalence of excessive daytime sleepiness $(88 \%)$, physically inactive $(38.2 \%)$, prehypertensive $(14.7 \%)$ SBP and $(27.1 \%)$ DBP. Regarding body mass index, females presented higher indices, but for waist and neck circumference, males presented higher indices $(p<0.05)$. Conclusion: The presence and positive associations between risk factors that may contribute to the development of cardiovascular diseases serve as indicators for preventive measures to be taken.

DESCRIPTORS: Risk factors, Lifestyle, Adolescents.

\section{RESUMEN}

Los factores de riesgo cardiovascular y los factores asociados pueden sumarse al desarrollo de enfermedades cardiovasculares. Objetivo:Analizar la frecuencia de factores de riesgo cardiovascular y factores asociados en adolescentes. Método: Estudio transversal realizado con 251 estudiantes de secundaria de la ciudad de Teresina (PI), entre 14 y 17 años. Se obtuvo información sobre estilo de vida, datos antropométricos y clínicos. Resultados: La edad promedio de los adolescentes fue de 16,2 $\pm 0,76$ años. Hubo una prevalencia de somnolencia diurna excesiva (88\%), inactividad física (38,2\%), PAS prehipertensos $(14,7 \%)$ y EAP $(27,1 \%)$. En cuanto al índice de masa corporal, las mujeres tuvieron tasas más altas, sin embargo, para la circunferencia de cintura y cuello, los hombres tuvieron tasas más altas $(p<0.05)$. Conclusión: La presencia y asociaciones positivas entre factores de riesgo que pueden contribuir al desarrollo de enfermedades cardiovasculares sirven como señales para la toma de medidas preventivas.

DESCRIPTORES: Factores de Riesgo; Estilo de Vida; Adolescentes.

RECEBIDO EM: 08/05/2021 APROVADO EM: 14/05/2021

\section{Fabiana Neves Lima}

Mestre em saúde e comunidade, PROFESSORA/IFPI.

ORCID: 0000-0002-1894-6529 


\section{Camila Aparecida Pinheiro Landim Almeida}

Doutora em enfermagem. Docente/Instituto de Ciências de Saúde da Universidade Católica Portuguesa (ICS-UCP), Porto, Portugal. ORCID: 0000-0003-4843-4572

\section{Regina Célia Vilanova Campelo}

Doutora. Universidade Estadual do Maranhão (UEMA).

ORCID: 0000-0002-3221-2927

\section{Luisa Helena de Oliveira Lima}

Doutorado em Enfermagem. UFPI/PICOS.

ORCID: 0000-0002-1890-859X

\section{Ana Roberta Vilarouca da Silva}

Doutora. Universidade Federal do Piauí (UFPI).

ORCID: 0000-0001-5087-4310

\section{Ana Larissa Gomes Machado}

Doutorado em Enfermagem. UFPI/PICOS.

ORCID: 0000-0002-7937-6996

\section{INTRODUÇÃO}

D entre as Doenças Crônicas Não Transmissíveis (DCNT), as Doenças Cardiovasculares (DCV) estão no topo com 31,3\%, a hipertensão, que é um Fator de Risco Cardiovascular (FRCV), afeta de $2 \%$ a $13 \%$ das crianças e adolescentes ${ }^{1}$. A prevalência das DCV é uma preocupação crescente considerada umas das maiores causas de morbimortalidade $^{2}$. Neste contexto, é importante o desenvolvimento de estratégias preventivas, pois estudos multicêntricos apresentam diferenças regionais de $\mathrm{FRCV}^{3}$.

A Organização Mundial de Saúde $(\mathrm{OMS})^{4}(2006)$ considera que a prática de atividade física previne ou reduz a hipertensão arterial, depressão, cardiopatias, sobrepeso entre outas enfermidades, além de promover bem-estar em seus praticantes. E esta de forma planejada proporciona diversas adaptações morfológicas e fisiológicas no sistema cardiovascular, melhorias no funcionamento do sistema cardiorrespiratório, dos índices de composição corporal, hipotensão pós-exercício e pós-treinamento ${ }^{4,5}$.

Na puberdade evidencia-se a adoção de comportamento de risco para a saúde, que tende a se manter durante a fase adulta, o que pode interferir na qualidade de vida ${ }^{6}$. Elicker et al. ${ }^{78}$ relatam o uso de drogas como uma preocupação mundial, e tanto álcool como tabaco com maiores taxas de
A prevalência

das DCV é uma

preocupação

crescente

considerada umas

das maiores causas de

morbimortalidade.

Neste contexto,

é importante o

desenvolvimento

de estratégias

preventivas,

pois estudos

multicêntricos

apresentam diferenças

regionais de FRCV. mortalidade no mundo. O uso precoce de álcool na adolescência está associado a problemas de saúde na fase adulta, contribuindo com o risco de consumo em excesso ao longo da vida ${ }^{16,17}$.

O hábito de sono é considerado comportamento benéfico para o organismo e essencial para o ciclo de 24 horas $^{8}$. Legnani ${ }^{9}$ descreve que bons níveis de sono estão associados a maiores frequências de comportamentos promotores de saúde, e a falta de sono ou mesmo níveis insuficientes de sono estão associados ao uso de tabaco e álcool. Nesse cenário, destaca-se a Sonolência Diurna Excessiva (SDE) relatada por Felden et at. ${ }^{10}$ como uma sensação aumentada da necessidade do sono, e diminuição no estado de alerta. $\mathrm{Na}$ adolescência observa-se um atraso na fase do sono caracterizado por estágios mais tardios para acordar, o que pode está associado a quadros de hipertensão, estudos demonstram associação entre má qualidade do sono tanto com menor quanto com excessivo tempo de sono e pressão arterial elevada $^{11,12,13,14}$.

Estudos dessa natureza contribuiem para elaboração das intervenções destinadas à promoção de estilo de vida saudável entre adolescentes, e auxiliará os profissionais de saúde com parâmetros confiáveis. Neste sentido, o estudo objetivou analisar a frequência de fatores de riscos cardiovasculares em adolescentes. 


\section{artigo}

Lima, F.N.; Almeida, C.A.P.L.; Campelo, R.C.V.; Lima, L.H.O.; Vilarouca da Silva, A.R.; Machado, A.L.G.;

Prevalência de risco cardiovascular e fatores associados em adolescentes

\section{MÉTODO}

Estudo Tranversal realizado com 251 estudantes de escolas públicas da regularmente matriculados entre o $1^{\circ}$ e $3^{\circ}$ anos do Ensino Médio de uma escola pública Teresina (Piauí, Brasil).

Os estudantes foram selecionados por amostragem probabilística aleatória simples através de sorteio realizado pelo programa BioEstat, versão 5.3 e estratificada por ano de ensino médio $\left(1^{\circ}, 2^{\circ}\right.$ e $3^{\circ}$ anos), e a amostra calculada com base na população finita e teste de normalidade.

O estudo foi aprovado pelo Comitê de Ética em Pesquisa da Universidade Federal do Piauí/UFPI-CCS sob CAA 90250618.3.0000.8057, sendo a mensuração de dados antropométricos, pressão arterial e a aplicação dos questionários com os escolares foram realizadas somente mediante devolução do Termo de Consentimento Livre Esclarecido pelos pais/ responsáveis dos alunos selecionados e
Termo de Assentimento assinado pelos escolares, conforme resolução 510/16.

Realizou-se a coleta de dados de setembro a novembro de 2018, no horário de estudo dos escolares por avaliadores devidamente treinados para ocupar uma sala dividida em estaçoes ( $1^{\text {a }}$ estação: Questionários; $2^{\mathrm{a}}$ : medida da pressão arterial; $3^{\mathrm{a}}$ : medidas antropométricas).

$\mathrm{Na}$ análise estatística foi usado o software estatístico Statistical Package for Social Sciences $\left(\right.$ IBM SPSS $^{\circ}$ ) para Windows', versão 23.0. A normalidade das variáveis foi verificada pelo teste Kolmogorov-Smirnov, $(p>0,05)$, as variáveis IMC, PAS, PAD apresentaram distribuição normal. Utilizou-se o teste t-Student para variáveis categórica dicotômica, e ANOVA para variável com 3 ou mais categorias. As variáveis $\mathrm{CC}$ e $\mathrm{CP}$ apresentaram dados não normais $(\mathrm{p}<0,05)$; para essas variáveis foram utilizados o teste Mann-Whitney, para variável categórica dicotômica, e Kruskall-Wallis, para variável com 3 ou mais categorias. A análise descritiva incluiu o cálculo da média, medianas, desvio padrão, intervalo interquartil, mínimos e máximos, para as variáveis quantitativas; e frequências, para as qualitativas e seus respectivos intervalos de confiança de $95 \%$ (IC95\%).

\section{RESULTADOS}

Quanto ao estilo de vida, $94 \%$ não am do estudo 251 adolescentes com idade média de 16,2 anos $( \pm 0,76)$, e $60,1 \%$ do sexo feminino. apresentaram comportamento sedentário durante a semana. Em relação ao nível de atividade física, 38,2\% foram classificados como inativos. Acerca do consumo de álcool, 8,4\% estavam em zona de risco moderada, $6,0 \%$ estudantes usavam tabaco, $20,0 \%$ tinham consumido álcool nos últimos 30 dias, 53,0\% experimentaram pela primeira vez aos 14 ou 15 anos e 20,0\% costumam fumar em casa de amigos(Tabela 1).

Tabela 1. Distribuição da amostra de adolescentes, em valores absolutos e proporcionais, de acordo com o Estilo de Vida, a percepção da qualidade do sono e sonolência diurna, Teresina - Piauí, 2019.

VARIÁVEIS

Comportamento sedentário na semana (CS)

$\operatorname{Sim}$

Não

Comportamento sedentário no fim de semana

$\operatorname{Sim}$

Não

Nivel de atividade física

Muito ativo

Moderadamente ativo

Inativo

Consumo de álcool

Baixo risco

Médio risco

Síndrome da dependência do álcool

Uso de tabaco

$\operatorname{Sim}$

Não

15

Nos últimos 7 dias, em quantos dias alguém fumou na sua presença 


\begin{tabular}{|c|c|c|}
\hline Nenhum dia & 124 & 49,3 \\
\hline 1 ou 2 dias & 74 & 29,5 \\
\hline 3 ou 4 dias & 23 & 9,2 \\
\hline 5 ou 6 dias & 04 & 1,6 \\
\hline Todos os 7 dias & 26 & 10,4 \\
\hline \multicolumn{3}{|c|}{ Consumo de tabaco pelos pais } \\
\hline Nenhum & 198 & 78,8 \\
\hline Pai & 30 & 12,0 \\
\hline Mãe & 12 & 4,8 \\
\hline Ambos & 01 & 0,4 \\
\hline Não sabe & 10 & 4,0 \\
\hline \multicolumn{3}{|c|}{ Consumo de tabaco pelos amigos } \\
\hline Não & 157 & 62,5 \\
\hline A maioria & 14 & 5,6 \\
\hline Poucos & 80 & 31,9 \\
\hline \multicolumn{3}{|c|}{ Com que frequência você considera que dorme bem } \\
\hline Nunca & 05 & 2,0 \\
\hline Raramente & 44 & 17,5 \\
\hline Algumas vezes & 93 & 37,1 \\
\hline A maioria das vezes & 80 & 31,9 \\
\hline Sempre & 29 & 11,6 \\
\hline \multicolumn{3}{|c|}{ Como você avalia a qualidade do seu sono } \\
\hline Ruim & 33 & 13,1 \\
\hline Regular & 96 & 38,2 \\
\hline Boa & 74 & 29,5 \\
\hline Muito boa & 31 & 21,4 \\
\hline Excelente & 17 & 6,8 \\
\hline \multicolumn{3}{|c|}{ Quantas horas de sono nos dias de semana } \\
\hline Menos de 6 horas & 60 & 23,9 \\
\hline 6 horas & 87 & 34,7 \\
\hline 7 horas & 54 & 21,5 \\
\hline 8 horas & 36 & 14,3 \\
\hline 9 horas & 09 & 3,6 \\
\hline 10 ou mais horas & 05 & 2,0 \\
\hline \multicolumn{3}{|c|}{ Quantas horas de sono nos dias de final de semana } \\
\hline Menos de 6 horas & 19 & 7,6 \\
\hline 6 horas & 25 & 10,0 \\
\hline 7 horas & 26 & 10,4 \\
\hline 8 horas & 63 & 25,1 \\
\hline 9 horas & 56 & 22,3 \\
\hline 10 ou mais horas & 62 & 4,7 \\
\hline
\end{tabular}




\section{artigo}

Lima, F.N.; Almeida, C.A.P.L.; Campelo, R.C.V.; Lima, L.H.O.; Vilarouca da Silva, A.R.; Machado, A.L.G.;

Prevalência de risco cardiovascular e fatores associados em adolescentes

Excesso de sonolência diurna

Não

Fonte: dados da pesquisa.

A Pressão arterial sistólica (PAS) média e a Pressao Arterial Diastólica (PAD) média do sexo masculino apresentaram valores médios superiores em relação ao sexo feminino $(110,8$ e 63,9$)$ respectivamente; contrário a estes dados a Frequencia Cardíaca (FC) do sexo feminino foi maior que do sexo masculino, em ambos $(\mathrm{p}<0,05)$. Sobre os níveis de atividade física, a PAS média dos adolescentes muito ativos apresentou médias maiores $(108,6)$ em relação às demais categorias, e os considerados inativos apresentaram maiores valores médios de FC $(77,5)$, ambos os resultados $(p<0,05)$. Na categoria alto risco ou uso nocivo de álcool, os adolescentes apresentaram valores médios elevados da PAS média $(\mathrm{p}<0,05)$. E apesar da alta prevalência de sonolência diurna, os adolescentes com pouca sonolência diurna apresentaram PAD média com níveis superiores (67) quando comparados aos com sonolência diurna excessiva $(\mathrm{p}<0,05)$. (Tabela 2$)$.

Tabela 2. Comparação de médias das variáveis antropométricas com os dados socioeconômicos e relacionados ao estilo de vida dos estudantes, Teresina - Piauí, 2019.

\begin{tabular}{|c|c|c|c|c|c|c|}
\hline \multirow{2}{*}{ VARIÁVEIS } & \multicolumn{2}{|c|}{ IMC } & \multicolumn{2}{|c|}{ CC } & \multicolumn{2}{|c|}{ CP } \\
\hline & $X \pm D P^{*}$ & P-VALOR & $\mathrm{X} \pm \mathrm{DP} *$ & P-VALOR & $\mathrm{X} \pm \mathrm{DP} *$ & P-VALOR \\
\hline Sexo & & $0,020^{t}$ & & $0,010^{m}$ & & $0,000^{m}$ \\
\hline Masculino & $20,0 \pm 3,0$ & & $70,3 \pm 6,8$ & & $34,8 \pm 4,0$ & \\
\hline Feminino & $21,0 \pm 3,2$ & & $68,3 \pm 7,5$ & & $30,4 \pm 1,9$ & \\
\hline Ano de estudo & & $0,426^{a}$ & & $0,512^{k}$ & & $0,856^{k}$ \\
\hline $1^{\circ}$ ano & $20,4 \pm 2,9$ & & $68,7 \pm 6,3$ & & $32,2 \pm 4,3$ & \\
\hline $2^{\circ}$ ano & $21,0 \pm 3,5$ & & $70,2 \pm 9,0$ & & $32,1 \pm 3,1$ & \\
\hline $3^{\circ}$ ano & $20,5 \pm 3,0$ & & $68,3 \pm 6,4$ & & $31,9 \pm 2,9$ & \\
\hline Classe econômica & & $0,880^{a}$ & & $0,162^{k}$ & & $0,002^{k}$ \\
\hline A & $19,8 \pm 2,7$ & & $66,4 \pm 5,8$ & & $30,0 \pm 5,0$ & \\
\hline B1 - B2 & $20,7 \pm 3,5$ & & $70,2 \pm 7,8$ & & $33,3 \pm 4,7$ & \\
\hline $\mathrm{C} 1-\mathrm{C} 2$ & $20,6 \pm 3,1$ & & $68,8 \pm 7,3$ & & $31,6 \pm 2,7$ & \\
\hline$D-E$ & $20,3 \pm 2,6$ & & $66,7 \pm 5,3$ & & $30,7 \pm 2,0$ & \\
\hline Nível de atividade física & & $0,390^{t}$ & & $0,096 k$ & & $0,000^{k}$ \\
\hline Muito ativo & $20,8 \pm 3,3$ & & $70,2 \pm 8,0$ & & $33,4 \pm 2,9$ & \\
\hline Moderadamente ativo & $20,1 \pm 2,7$ & & $67,8 \pm 6,0$ & & $31,8 \pm 5,2$ & \\
\hline Inativo & $20,7 \pm 3,3$ & & $68,9 \pm 7,4$ & & $31,0 \pm 2,2$ & \\
\hline Consumo de álcool & & $0,028^{a}$ & & $0,008^{k}$ & & $0,065^{k}$ \\
\hline Baixo risco & $20,4 \pm 3,1$ & & $68,7 \pm 7,2$ & & $31,9 \pm 2,9$ & \\
\hline Médio risco & $22,6 \pm 4,0$ & & $73,0 \pm 7,4$ & & $33,9 \pm 7,8$ & \\
\hline Alto risco ou uso nocivo & $21,1 \pm 2,5$ & & $74,0 \pm 4,1$ & & $35,7 \pm 1,1$ & \\
\hline Síndrome da dependência & $20,1 \pm 1,2$ & & $62,7 \pm 1,7$ & & $29,8 \pm 0,8$ & \\
\hline Uso de tabaco & & $0,141^{t}$ & & $0,601^{m}$ & & $0,367^{\mathrm{m}}$ \\
\hline Sim & $21,8 \pm 2,7$ & & $71,0 \pm 7,6$ & & $31,5 \pm 3,0$ & \\
\hline Não & $20,5 \pm 3,2$ & & $68,9 \pm 7,3$ & & $32,1 \pm 3,6$ & \\
\hline Sonolência diurna & & $0,286^{t}$ & & $0,899^{m}$ & & $0,412^{m}$ \\
\hline
\end{tabular}



Excesso de sonolência
$20,7 \pm 3,2$
$69,1 \pm 7,5$
$32,1 \pm 3,7$
Pouca sonolência
$20,0 \pm 2,9$
$68,6 \pm 6,0$
$32,2 \pm 2,4$
*X土DP: média \pm desvio-padrão; ${ }^{\mathrm{t}}$ Teste t-Student / a ANOVA / m Mann-Whitney /k Kruskal-Wallis; valores significativos ( $\left.\mathrm{p} \leq 0,05\right)$ estão destacados em negrito. Fonte: dados da pesquisa.

\section{DISCUSSÃO}

A promoção da saúde na escola é uma preocupação mundial pois, é na adolescência que são incorporados hábitos de vida que podem persistir até a fase adul$\mathrm{ta}^{45,46,47}$, contribuindo para o desencadeamento de fatores de risco cardiovasculares que têm sido associados a um acentuado risco de morbidade e mortalidade ${ }^{4,8}$.

$\mathrm{O}$ estudo avaliou os fatores de riscos cardiovasculares (FRCV) como o estilo de vida dos adolescentes, considerando a inatividade física, o consumo de álcool e tabaco e o sono. Para obesidade foram estabelecidos os dados do índice de massa corpórea (IMC), circunferência da cintura (CC) e circunferência do pescoço (CP), e os clínicos a verificação da hipertensão através da PAS e PAD.

Foram identificados 236 (94\%) adolescentes que não apresentaram CS durante a semana. Esse resultado pode ser explicado pelo sistema de Ensino Integral, em que os alunos estão inseridos pois esses permanecem oito horas na escola estando restritos para utilização de celulares, computador, TV e videogame. Contudo, no final de semana esses apresentaram uma pequena elevação do CS resultado que corrobora com similares ${ }^{15}$ que denotam para importância de manter atividade física no ambiente escolar ${ }^{16}$.

Acerca da qualidade do sono $38,2 \%$ consideravam regular. Estudos apontam a associação entre curta duração do sono e a má qualidade do sono entre os adolescentes $^{16,17,18,19}$. A National Sleep Foundation $^{21}$ denota ponto de corte para o tempo de sono em adolescentes um período que pode variar de $8 \mathrm{~h}$ a $10 \mathrm{~h}$. Desta forma percebe-se que parte dos adolescentes do presente estudo está abaixo deste ponto de corte.

Os adolescentes tiveram prevalência de eutrofia para classificação do IMC, corro-
O estudo avaliou

os fatores de riscos

cardiovasculares

(FRCV) como

o estilo de vida

dos adolescentes,

considerando a

inatividade física, 0

consumo de álcool

e tabaco e o sono.

Para obesidade

foram estabelecidos

os dados do índice

de massa corpórea

(IMC), circunferência

da cintura (CC) e

circunferência do

pescoço (CP), e os

clínicos a verificação

da hipertensão através

da PAS e PAD. borando outros estudos que apresentaram maiores percentuais de eutrofia entre os adolescentes 62,8\%, 66,2\% respectivamente, ${ }^{4,5}$. Entretanto, estudo realizado por Brito et al. ${ }^{14}$ mostrou que tanto o sexo feminino quanto o masculino apresentaram níveis de sobrepeso e obesidade maiores. Mas o IMC entre os sexos apresentou diferença estatística, onde o sexo feminino apresentou IMC superior ao sexo masculino. $\mathrm{O}$ estudo da $\mathrm{PeNSE}^{17}$ afirma que níveis mais elevados tendem a ter maior prevalência entre o sexo feminino.

A obesidade abdominal foi determinada em 4,8\% da amostra. Valores similares foram encontrados em outros estudos com adolescentes ${ }^{5,8}$, ao comparar a variável entre os sexos a CC do sexo masculino foi significativamente maior que o sexo feminino. Outros estudos encontraram diferenças semelhantes entre os $\operatorname{sexos}^{22,23,24}$. Também a CP no sexo masculino foi significativamente maior que no feminino. Outros estudos encontraram valores semelhantes ${ }^{19,24}$, e ao realizar comparações entre a CP e as classes B1-B2, essas apresentaram valores elevados.

Assim pressupõem que menores níveis de consumo de álcool estiveram associados a menores índices antropométricos, o que pode ser considerado fator de proteção.

Os adolescentes mais ativos apresentaram PAS média superior às demais categorias, um estudo encontrou valores similares, entretanto sem diferenças significativas66. Apesar dessa diferença níveis altos de atividade física estão relacionados a baixas prevalências de $\mathrm{HAS}^{18,22}$ Os efeitos do exercício físico na diminuição da PA e a ações e mecanismos desta resposta não parece ser ainda uma afirmação aceita de forma majoritária, principalmente no público adolescente ${ }^{66}$.

O estudo teve limitação devido viés de memória, que foi corrigido pela utilização de questionários validados. 


\section{CONCLUSÃO}

O trabalho permite compreender o modo como a frequência de fatores de risco cardiovasculares se manifesta em diferentes contextos da vida do adolescente, apoiando futuras intervenções, ou ainda, fornecendo uma base para o monitoramento de tendências, assim promovendo um estilo de vida saudável neste público.

\section{REFERÊNCIAS}

1. Ministério da Saúde (BR). Secretaria de Vigilância em Saúde. Departamento de Análise de Situação de Saúde. [Internet]. Plano de ações estratégicas para o enfrentamento das doenças crônicas não transmissiveis (DCNT) no Brasil 2011 -2012. 2011 [acessado 2019 Mar 24]. Disponivel em: http://actbr.org.br/uploads/ conteudo/918_cartilha_dent.pdf. Acesso 17 abril. 2017

2. Garofolo L, Ferreira SRG, Miranda Junior F. Study of risk factors associated with Peripheral Arteriopathy in Japanese-Brazilians from Bauru (SP). Arq Bras Cardiol 2014; 102 (2):143-150.

3. Conselho Nacional de Saúde. (2016). Resolução n 510/2016. Recuperado em 31 de outubro de 2021.

4. Bloch KV, Cardoso MA, Sichieri R. Estudo dos Riscos Cardiovasculares em Adolescentes (ERICA): resultados e potencialidade. Rev Saúde Pública 2016; 50 (Supl.2):3S.

5. World Health Organization. Child Growth Standards: length/ height-for-age, weightforage,weight-for-length, weight-forheight and body mass index-for-age. Methods and development. Geneva, 2006. [acessado 2019 Mar 25] Disponível em: http:// www.who.int/childgrowth/en/

6. Brand C, Griebeler LC, Roth MA, Mello FF, Barros TVP, Neu LD. Effect of Resistance Training on Cardiovascular Parameters in Normotensive and Hypertensive Adults. Rev Bras Cardiol 2013; 26(6): 435-441.

7. Brum PC, Forjaz CLM, Tinucci, T, Negrão, C. E. Adaptações agudas e crônicas do exercício físico no sistema cardiovascular. Rev Paul Educ Fís 2004; 18(esp): 21-31.

8. Brito, ALS, Hardman CM, Barros MVG. Prevalência e fatores associados à simultaneidade de comportamentos de risco à saúde em adolescentes. Rev Paul Pediatr 2015; 33(4): 423-430.

9. Elicker E, Palazzo LS, Aerts DRGC, Alves GG, Câmara S. Uso de álcool, tabaco e outras drogas por adolescentes escolares de Porto Velho-RO, Brasil. Epidemiol Serv Saúde [online] 2015; 24(3); 399-410.

10. Strauch ES, Pinheiro RT, Silva RA, Horta BL. Uso de álcool por adolescentes: estudo de base populacional. Revista de Saúde Pública 2009; 43( 4): 647-655.

11. Instituto Brasileiro de Geografia e Estatística (IBGE). Pesquisa Nacional de Saúde do Escolar 2015. Rio de Janeiro: IBGE; 2015.

12. Legnani RFS. Elaboração, validação e aplicação de um Questionário via web para avaliar os comportamentos relacionados à saúde em escolares. [tese] Paraná: Universidade Federal do Paraná; 2015.

13. Felden EPG, Filipin D, Barbosa DG, Andrade RD, Meyer C, Beltrame TS. Adolescentes com sonolência diurna excessiva passam mais tempo em comportamento sedentário. Rev Bras Med Es- porte 2016; 22(3): 186-190.

14. Gottlieb D J, Redline S, Nieto FJ, Baldwin CM, Newman AB, Resnick HE, Punjabi NM. Association of Usual Sleep Duration With Hypertension: The Sleep Heart Health Study. SLEEP 2006; 29(8).

15. Hannon TS, Tu W, Watson SE, Jalou H, Chakravorty S, Arslanian SA. Morning Blood Pressure is Associated with Sleep Quality in Obese Adolescents. J Pediatr 2014; 164(2): 313-317.

16. Gonzaga NC, Sena ASS, Coura AS, Dantas FG, Oliveira RC. Sleep quality and metabolic syndrome in overweight or obese children and adolescents. Rev Nutr 2016; (29)3: 377-389.

17. Paciência I, Araújo J, Ramos E. Sleep duration and blood pressure: a longitudinal analysis from early to late adolescence. J Sleep Res 2016; 25: 702-708.

18. Carvalho FFB. A saúde vai a escola: a promoção da saúde em práticas pedagógicas. Physis 2015; 25(4): 1207-1227.

19. Brasil EGM, Silva RM, Silva MRF, Rodrigues DP, Queiroz MVO. Promoção da saúde de adolescentes e Programa Saúde na Escola: complexidade na articulação saúde e educação. Rev Esc Enferm 2017; 51.

20. Barbosa FNM, Casotti CA, Nery, A. A. Comportamento de risco a saúde de adolescentes escolares. Texto Contexto Enferm 2016; 25(4): 1-9.

21. Mazzardo O, Silva MP da, Guimarães RF, Martins RV, Watabe $\mathrm{Pl}$, Campos W. Comportamentos de risco a saúde entre adolescentes de acordo com gênero, idade e nível socioeconômico. Medicina 2016; 49(4): 321-330.

22. Raphaelli CO, Pretto ADB, Dutra EF. Prevalência de hábitos de vida em escolares de um Município do Sul do Brasil. Adolesc Saude 2016; 13(2): 16-23.

23. Lund $H G$, Reider $B D$, Whiting AB, Prichard JR. Sleep patterns and predictors of disturbed sleep in a large population of college students. J Adolesc Health 2010; 46(2): 124-132.

24. Pereira EF, Barbosa DG, Andrade RD, Claumann GS, Pelegrini A, Louzada EM. Sono e adolescência: quantas horas os adolescentes precisam dormir. J Bras Psiquiatr 2015; 64(1): 40-44.

25. Meyer C, Ferrari Júnior GJ, Barbosa DG, Andrade RD, Pelegrini A, Felden EPG. Análise da sonolência diurna em adolescentes por meio da pediatric daytime sleepiness scale: revisão sistemática. Rev Paul Pediatr 2017; 35(3): 351-360.

26. da Silva Gouveia Lima , A. ., Silveira Ribeiro, K. C. ., Virginia Silva de Sousa, V. Laurentino Barbosa , A. ., da Silva Bezerra de Lima, R. ., \& Renaly Mota Rodrigues, A. . (2020). A utilização de álcool na população adolescente e sua relação com ideias suicídas. Saúde Coletiva (Barueri), 10(58), 4053-4066. https://doi.org/10.36489/ saudecoletiva.2020v10i58p4053-4066 